\title{
On the linear theory of Kelvin-Helmholtz instabilities of relativistic magnetohydrodynamic planar flows
}

\author{
Z. Osmanov ${ }^{1}$, A. Mignone ${ }^{1,2}$, S. Massaglia ${ }^{1}$, G. Bodo ${ }^{2}$, and A. Ferrari ${ }^{1}$ \\ 1 Dipartimento di Fisica Generale, Universitá degli Studi di Torino, via Pietro Giuria 1, 10125 Torino, Italy \\ e-mail: z.osmanov@astro-ge.org; osmanov@ph.unito.it \\ 2 INAF/Osservatorio Astronomico di Torino, Strada Osservatorio 20, 10025 Pino Torinese, Italy
}

Received 19 February 2008 / Accepted 1 August 2008

\section{ABSTRACT}

\begin{abstract}
Aims. We investigate the linear stability properties of the plane interface separating two relativistic magnetized flows in motion with respect to each other. The two flows are governed by the (special) relativistic equations for a magnetized perfect gas in the infinite conductivity approximation.

Methods. By adopting the vortex-sheet approximation, the relativistic magnetohydrodynamics equations are linearized around the equilibrium state and the corresponding dispersion relation is derived and discussed. The behavior of the configuration and the regimes of instability are investigated following the effects of four physical parameters: the flow velocity, the relativistic and Alfvénic Mach numbers, and the inclination of the wave vector on the plane of the interface.

Results. From the numerical solution of the dispersion relation, we find in general two separate regions of instability, associated with the slow and fast magnetosonic modes respectively. Modes parallel to the flow velocity are destabilized only for sufficiently low magnetization. For the latter case, stabilization is attained, in addition, at sufficiently large relativistic velocities between the two flows in relative motion.

Conclusions. We briefly comment the relevance of these results to the study of the stability of astrophysical jets.
\end{abstract}

Key words. magnetohydrodynamics (MHD) - instabilities - plasmas

\section{Introduction}

Acting at the contact interface between two flows in motion with respect to each other the Kelvin-Helmholtz instability (hereafter KHI), plays a dynamically important role in a number of different astrophysical scenarios, such as accretion disks, planetary magnetospheres, and stellar and extragalactic jets. It is a classical instability in fluid dynamics, which was discussed at the end of nineteenth century by Von Helmholtz \& Monats (1868) and Lord Kelvin (1871). The classical results of the linear analysis for incompressible flows separated by a planar vortex sheet, both in the presence of magnetic fields and for different geometries, were summarized in Chandrasekhar's monograph (Chandrasekhar 1961). They were later generalized to the compressible case, both at the pure hydrodynamical (see e.g. Gervin 1968; and Sen 1964) and the magnetohydrodynamic (MHD) limits (see e.g., Sen 1963; and Pu \& Kivelson 1983), extending the study to the supersonic regime. In particular, it was shown that, in the hydrodynamic case, no unstable longitudinal modes (with respect to the plane interface) are allowed for flow Mach numbers higher than $\sqrt{8}$, while transverse modes are always unstable.

Choudhury \& Lovelace (1986) studied the linear instability behavior in the case of a finite-thickness boundary layer, as a function of the Alfvèn velocity and inclination of the wave vector with respect to the interface. Their main result was the proof of the existence of two distinct regimes of instability in the wavevector-Mach number plane, which corresponded to standing and traveling MHD waves, the extent of the instability regions depending upon the magnetization parameter.
Magnetohydrodynamic flows in cylindrical and slab geometries were studied by Ferrari et al. (1981, 1982) indicating a new range of unstable modes arising for high Mach number flows, due to standing waves created by reflections at the boundaries of the structures.

For astrophysical problems involving high-energy phenomena relativistic effects become important either because the relative velocities are close to the speed of light, the plasma is relativistically hot, or the magnetic field is very strong and the Alfvèn speed becomes close to the speed of light. For the nonmagnetic case, a relativistic extension of the KHI study was considered, for a plane-parallel velocity discontinuity, by Turland \& Scheuer (1976), Blandford \& Pringle (1976), and Bodo et al. (2004). Bodo et al. (2004) demonstrated that the dispersion relation can be solved analytically in a frame of reference in which the media in the two half-spaces have equal and opposite velocities, and that the stability criteria have the same form as classical ones, after substituting the Mach number with the relativistic Mach number, first introduced by Königl (1980).

The relativistic MHD case has not been studied by a full stability analysis, which we present in this paper. Early attempts were carried out by Ferrari et al. (1980). Their analysis, however, assumed a limit of the Alfv 'en velocity, which is far smaller than the speed of light; this allows displacement currents to be neglected, although the flow speed can reach relativistic values. The main result of this study was the confirmation that KHI disappears when the Alfvèn velocity approaches or becomes bigger than the speed of sound and when a high density contrast exists between the two media. 
Relativistic magnetized flows can be found in astrophysical environments associated with AGN jets, to jets from galactic X-ray binaries, such as GRS 1915+105 (Mirabel \& Rodriguez 1999), and pulsar wind nebulae (PWNe). Applications of the Kelvin-Helmholtz instability mechanism to the case of AGN and galactic microquasar jets is discussed mainly for cylindrical geometries, such as by Hardee (2007), who studied axially magnetized cylindrical relativistic jet embedded in a magnetized sheath. In addition, KHI appear to be the physical mechanism that decelerate relativistic jets by producing entrainment of the ambient medium. Bodo et al. (2003), Rossi et al. (2004), and Rossi et al. (2008) studied the nonlinear evolution of KHI in cylindrical relativistic jets to explain the dichotomy in the properties of FRI-FRII extragalactic radio sources. The planar case applies instead to the case of PWNe, when a relativistically hot magnetized plasma forms and the pulsar ultra-relativistic wind interacts with the surrounding supernova ejecta. The linear stability analysis that we present is relevant to understanding the physical phenomena involved in the destabilization process.

For the first time to our knowledge we present, a complete treatment of the linear stability problem in the plane-parallel geometry, by considering the full system of equations i.e. without neglecting the displacement current and accounting for nonrelativistic as well as relativistic Alfvèn velocities. It is shown that the stability properties depend on four physical parameters, which we conveniently choose to be the relativistic Mach number, the flow velocity, the Alfvèn speed, and the inclination of the projection of the wave vector onto the plane of the interface.

The paper is organized as follows. In Sect. 2 we derive the general dispersion relation, in Sect. 3 we present and discuss the results for the non-relativistic and relativistic cases, and we add some astrophysical comments in Sect. 4.

\section{Relevant equations}

\subsection{The equations of relativistic MHD}

The equations of relativistic magnetohydrodynamics (hereafter RMHD) govern the evolution of a (special) relativistic magnetized fluid. They can be cast as a system of conservation laws, describing energy-momentum and mass conservation:

$\nabla_{\mu} T^{\mu v}=0, \quad \nabla_{\mu}\left(\rho u^{\mu}\right)=0$,

where $\rho$ is the rest mass density and $u^{\mu} \equiv(\gamma, \gamma \boldsymbol{v})$ is the fluid four-velocity ( $\gamma \equiv$ Lorentz factor, $\boldsymbol{v} \equiv$ three velocity) such that $u^{\mu} u_{\mu}=-1$. The speed of light is set to be unity everywhere. The expression of the energy-momentum tensor can be found under the physical assumptions of constant magnetic permeability and infinite conductivity, appropriate for a perfectly conducting fluid (see, for example, the books by Anile 1989 and Lichnerowitz 1967).

Under these conditions, the stress energy tensor for a perfect fluid interacting with an electromagnetic field decomposes into $T^{\mu \nu}=T_{\mathrm{FL}}^{\mu \nu}+T_{\mathrm{EM}}^{\mu \nu}$, where the fluid (FL) and electromagnetic (EM) contributions are given, respectively, by

$T_{\mathrm{FL}}^{\mu v}=\rho h u^{\mu} u^{v}+p \eta^{\mu \nu}, T_{\mathrm{EM}}^{\mu \nu}=F_{\beta}^{\mu} F^{\nu \beta}-\frac{1}{4} \eta^{\mu \nu} F_{\alpha \beta} F^{\alpha \beta}$.

The symbols $h$ and $p$ represent the gas specific enthalpy and the thermal pressure, while $\eta^{\mu \nu}=\operatorname{diag}(-1,1,1,1)$ is the Minkowski metric tensor. For ease of notation, such as $4 \pi$ have been set to unity in the derivation.
The electromagnetic field tensor $F^{\mu v}$ appearing in the definitions of the stress energy tensor, Eq. (2), obeys Maxwell's equations

$\partial_{[\alpha} F_{\mu v]}=0, \quad \nabla_{\mu} F^{\mu v}=-J^{v}$,

where $[\ldots]$ denotes anti-symmetrization and $J^{v}$ is the charge four-current. In the limit of infinite conductivity, the rest-frame electric field vanishes identically and the electromagnetic field tensor becomes orthogonal to the fluid four-velocity, i.e. $F^{\mu \beta} u_{\beta}=$ 0 . We note that this is the only approximation introduced in ideal RMHD and it becomes identical to the well known nonrelativistic expression $\boldsymbol{E}=-\boldsymbol{v} \times \boldsymbol{B}$, where $\boldsymbol{E}$ is the electric vector field. Unlike classical MHD, however, the displacement current is not discarded in RMHD and explicitly enters through the definition of the current, given by the second of Eqs. (3). The high conductivity limit allows us to write $F^{\mu v}=\epsilon^{\alpha \beta \mu \nu} b_{\mu} u_{v}$, where $b^{\mu}$ is the magnetic induction four-vector:

$b^{\mu}=\left[\gamma \boldsymbol{v} \cdot \boldsymbol{B}, \frac{\boldsymbol{B}}{\gamma}+\gamma(\boldsymbol{v} \cdot \boldsymbol{B}) \boldsymbol{v}\right]$

with $\boldsymbol{B}$ and $\epsilon^{\alpha \beta \mu \nu}$ denoting the rest-frame magnetic field and the Levi-Civita pseudo-tensor, respectively.

For the purpose of our derivation, we explicitly rewrite Eq. (1) in components:

$\frac{\partial \boldsymbol{U}}{\partial t}+\sum_{i=1}^{3} \frac{\partial \boldsymbol{f}^{i}}{\partial x^{i}}=0$

where $\boldsymbol{U}$ and $\boldsymbol{f}^{i}$ are the vector of conserved variables and corresponding fluxes:

$\boldsymbol{U}=\left[\rho \gamma, w_{\mathrm{t}} \gamma^{2} v^{j}-b^{0} b^{j}, w_{\mathrm{t}} \gamma^{2}-b^{0} b^{0}-p_{\mathrm{t}}\right]^{T}$,

$\boldsymbol{f}^{i}=\left[\rho \gamma v^{i}, w_{\mathrm{t}} \gamma^{2} v^{i} v^{j}-b^{i} b^{j}+p_{\mathrm{t}} \delta^{i j}, w_{\mathrm{t}} \gamma^{2} v^{i}-b^{0} b^{i}\right]^{T}$,

where $p_{\mathrm{t}}$ and $w_{\mathrm{t}}$ are the total pressure and enthalpy, respectively, expressed as the sum of the thermal and magnetic contributions:

$p_{\mathrm{t}}=p+\frac{1}{2}|b|^{2}, \quad w_{\mathrm{t}}=\rho h+|b|^{2}$,

where $|b|^{2}=b^{\alpha} b_{\alpha}$. Magnetic field evolution is given by the induction equation in Maxwell's law:

$\frac{\partial \boldsymbol{B}}{\partial t}-\nabla \times(\boldsymbol{v} \times \boldsymbol{B})=0$,

which has the same form as in the classical case. Proper closure is provided by specifying an equation of state, which we assume to be the constant $\Gamma$-law:

$h=1+\frac{\Gamma}{\Gamma-1} \frac{p}{\rho}$

where $\Gamma$ is the polytropic index of the gas.

\subsection{Dispersion relation}

Our setup consists of a planar vortex sheet interface in the $x z$ plane at $y=0$ separating two uniform flows moving in the $x$ direction with opposite velocities $\boldsymbol{v}(y>0)=\beta \boldsymbol{i}$ and $\boldsymbol{v}(y<0)=-\beta \boldsymbol{i}$. The fluids have equal density and pressure and are threaded by a uniform longitudinal magnetic field along the direction of relative motion, $\boldsymbol{B}=B_{0} \boldsymbol{i}$. 
We start our analysis by introducing small deviations about the equilibrium state. This is most efficiently completed in the fluid rest frame, in which we can model perturbations of the following forms:

$\tilde{\boldsymbol{B}}=\tilde{\boldsymbol{B}}_{0}+\tilde{\boldsymbol{B}}^{\prime}+\ldots$,

$\tilde{\boldsymbol{v}}=\tilde{\boldsymbol{v}}^{\prime}+\ldots$,

$\tilde{\rho}=\tilde{\rho}_{0}+\tilde{\rho}^{\prime}+\ldots$,

$\tilde{p}=\tilde{p}_{0}+\tilde{p}^{\prime}+\ldots$

where the tilde denotes quantities in the rest frame. The perturbed terms of the first order can be expressed by the following:

$\tilde{\Psi}_{ \pm}^{\prime} \propto \exp \left[i\left(\tilde{k}_{ \pm} \tilde{x}+\tilde{l}_{ \pm} \tilde{y}+\tilde{m}_{ \pm} \tilde{z}-\tilde{\omega}_{ \pm} \tilde{t}\right)\right]$

where $\tilde{\Psi}_{ \pm}^{\prime}=\left(\tilde{\boldsymbol{B}}_{ \pm}^{\prime}, \tilde{\boldsymbol{v}}_{ \pm}^{\prime}, \tilde{\rho}_{ \pm}^{\prime}, \tilde{p}_{ \pm}^{\prime}\right)$. Subscripts + and - correspond to the regions $y>0$ and $y<0$, respectively. Wave numbers and frequency are denoted by $\tilde{k}_{ \pm}, \tilde{l}_{ \pm}, \tilde{m}_{ \pm}$, and $\tilde{\omega}_{ \pm}$.

Proper linearization of the equations produces the dispersion relation Komissarov (1999):

$$
\begin{aligned}
& \left(\tilde{\omega}_{ \pm}^{2}-\tilde{k}_{ \pm}^{2} V_{A}^{2}\right) \times\left(\frac{\tilde{\omega}_{ \pm}^{4}}{\left(\tilde{k}_{ \pm}^{2}+\tilde{l}_{ \pm}^{2}+\tilde{m}_{ \pm}^{2}\right)^{2}}\right. \\
& \left.+\frac{\tilde{\omega}_{ \pm}^{2} \tilde{\mu}_{ \pm}}{\tilde{k}_{ \pm}^{2}+\tilde{l}_{ \pm}^{2}+\tilde{m}_{ \pm}^{2}}+\tilde{v}_{ \pm}\right)=0, \\
& \tilde{\mu}_{ \pm}=C_{\mathrm{s}}^{2}+V_{\mathrm{A}}^{2}-C_{\mathrm{s}}^{2} V_{\mathrm{A}}^{2} \frac{\tilde{k}_{ \pm}^{2}+\tilde{l}_{ \pm}^{2}}{\tilde{k}_{ \pm}^{2}+\tilde{l}_{ \pm}^{2}+\tilde{m}_{ \pm}^{2}}, \\
& \tilde{v}_{ \pm}=C_{\mathrm{s}}^{2} V_{\mathrm{A}}^{2} \frac{\tilde{k}_{ \pm}^{2}}{\tilde{k}_{ \pm}^{2}+\tilde{l}_{ \pm}^{2}+\tilde{m}_{ \pm}^{2}}, \\
& C_{\mathrm{s}}=\sqrt{\frac{\Gamma p_{0}}{\rho_{0} h_{0}}}, \\
& V_{\mathrm{A}}=\frac{B_{0}}{\sqrt{\rho_{0} h_{0}+B_{0}^{2}}},
\end{aligned}
$$

which allow the propagation of the Alfvèn mode

$$
\frac{\tilde{\omega}_{\mathrm{A} \pm}^{2}}{\tilde{k}_{\mathrm{A} \pm}^{2}}=V_{\mathrm{A}}^{2},
$$

and the two magneto-acoustic modes, (i) slow magnetosonic

$\frac{\tilde{\omega}_{\mathrm{s} \pm}^{2}}{\tilde{k}_{\mathrm{s} \pm}^{2}+\tilde{l}_{\mathrm{s} \pm}^{2}+\tilde{m}_{\mathrm{s} \pm}^{2}}=\frac{1}{2} \tilde{\mu}_{\mathrm{s} \pm}-\frac{1}{2}\left[\tilde{\mu}_{\mathrm{s} \pm}^{2}-4 \tilde{v}_{\mathrm{s} \pm}^{2}\right]^{1 / 2}$,

and (ii) fast magnetosonic

$$
\frac{\tilde{\omega}_{\mathrm{f} \pm}^{2}}{\tilde{k}_{\mathrm{f} \pm}^{2}+\tilde{l}_{\mathrm{f} \pm}^{2}+\tilde{m}_{\mathrm{f} \pm}^{2}}=\frac{1}{2} \tilde{\mu}_{\mathrm{f} \pm}+\frac{1}{2}\left[\tilde{\mu}_{\mathrm{f} \pm}^{2}-4 \tilde{v}_{\mathrm{f} \pm}^{2}\right]^{1 / 2},
$$

where $C_{\mathrm{S}}$ and $V_{\mathrm{A}}$ are the sound speed and Alfvèn speed, respectively. We note that both $C_{\mathrm{s}}$ and $V_{\mathrm{A}}$ are invariant under Lorentz boosts in the $x$-direction.

To obtain the dispersion relation in the laboratory frame, we have to transform all the quantities in terms of a Lorentz transformation:

$\tilde{\omega}_{ \pm}=\gamma(\omega \mp k \beta)$, $\tilde{k}_{ \pm}=\gamma(k \mp \omega \beta), \quad \tilde{l}_{ \pm}=l_{ \pm}, \quad \tilde{m}_{ \pm}=m$,

so that a generic perturbation in our original frame can be expressed as

$\Psi_{ \pm}^{\prime} \propto \exp \left[i\left(k x+l_{ \pm} y+m z-\omega t\right)\right]$.

By substituting Eqs. (24) and (25) into Eq. (21), we derive immediately that the possible roots are always real. We may therefore conclude that the Alfvèn mode does not contribute to the instability. In the case of Eqs. (22) and (23), we can see instead that the resulting slow and fast magnetosonic modes include both real and imaginary roots, and therefore we expect instability in the parameter domains corresponding to these modes.

Direct substitution into the induction equation Eq. (9) and into the $y$ and $z$ components of the momentum equation in Eq. (5) yields

$B_{0} \frac{\tilde{k}_{\mp}}{\gamma} B_{y \pm}^{\prime}+\left[\omega B_{0}^{2}+\gamma \rho_{0} h_{0} \tilde{\omega}_{\mp}\right] v_{y \pm}^{\prime}-l_{ \pm}\left(p_{ \pm}^{\prime}+B_{0} B_{x \pm}^{\prime}\right)=0$,

$B_{0} \frac{\tilde{k}_{\mp}}{\gamma} B_{z \pm}^{\prime}+\left[\omega B_{0}^{2}+\gamma \rho_{0} h_{0} \tilde{\omega}_{\mp}\right] v_{z \pm}^{\prime}-m\left(p_{ \pm}^{\prime}+B_{0} B_{x \pm}^{\prime}\right)=0$,

$(\omega \mp k \beta) B_{x \pm}^{\prime}-B_{0}\left(l_{ \pm} v_{y \pm}^{\prime}+m v_{z \pm}^{\prime}\right)=0$,

$(\omega \mp k \beta) B_{y \pm}^{\prime}+k B_{0} v_{y \pm}^{\prime}=0$,

$(\omega \mp k \beta) B_{z \pm}^{\prime}+k B_{0} v_{z \pm}^{\prime}=0$,

where $\tilde{k}_{\mp}$ and $\tilde{\omega}_{\mp}$ are given by Eqs. (24), (25).

With the aid of Eq. (8) one may express the total pressure perturbation as:

$p_{ \pm}^{t}=p_{ \pm}+\frac{l_{ \pm} B_{0}^{2} v_{y \pm}^{\prime}}{\omega \mp k \beta}$,

and imposing the displacement matching condition at the interface:

$\frac{v_{y+}^{\prime}}{\omega-k \beta}=\frac{v_{y-}^{\prime}}{\omega+k \beta}$

and the total pressure continuity $\left(p_{+}^{t}=p_{-}^{t}\right)$ at the interface, one obtains (together with Eq. (27))

$\frac{l_{+}}{l_{-}}=\frac{\rho_{0} h_{0} \gamma^{2}(\omega-k \beta)^{2}+\left(\omega^{2}-k^{2}\right) B_{0}^{2}}{\rho_{0} h_{0} \gamma^{2}(\omega+k \beta)^{2}+\left(\omega^{2}-k^{2}\right) B_{0}^{2}}$.

Introducing the dispersion relation of slow and fast magnetosonic waves (see the right bracket of Eq. (16)) and solving for $l_{ \pm}^{2}$, we obtain

$l_{ \pm}^{2}=-m^{2}+\frac{\tilde{\omega}_{\mp}^{2}\left[\left(C_{\mathrm{s}}^{2}+V_{\mathrm{A}}^{2}\right) \tilde{k}_{\mp}^{2}-\tilde{\omega}_{\mp}^{2}\right]-C_{\mathrm{s}}^{2} V_{\mathrm{A}}^{2} \tilde{k}_{\mp}^{4}}{C_{\mathrm{s}}^{2} V_{\mathrm{A}}^{2}\left(\tilde{k}_{\mp}^{2}+\tilde{\omega}_{\mp}^{2}\right)-\left(C_{\mathrm{s}}^{2}+V_{\mathrm{A}}^{2}\right) \tilde{\omega}_{\mp}^{2}}$,

which, combined with Eq. (34), provides the desired dispersion relation of modes that connect through the planar interface.

A number of considerations can be immediately drawn.

- The dispersion relation obtained by substitution of Eq. (34) into Eq. (35) is an 8-th degree polynomial in $\omega$. However, only 4 out of a total of 8 complex roots satisfy Eq. (34) and are therefore physically significant. 
- Roots with positive imaginary part of $\omega$ identify unstable modes. The growth rate will be more conveniently expressed by the imaginary part of the dimensionless quantity

$\phi \equiv \frac{\omega}{C_{\mathrm{s}} \sqrt{k^{2}+m^{2}}}$.

- In the non-relativistic limit (i.e. $\beta \rightarrow 0, \rho h \gg p, B_{0}^{2}$ and $h \rightarrow 1$ ), our dispersion relation reduces to the form given by Choudhury \& Lovelace (1986) in their equations Eqs. (7) and (A2a).

- In the limit of a vanishing magnetic field, Eqs. (34) and (35) simplify considerably and the dispersion relation reduces to the one derived by Bodo et al. (2004) (see their Eqs. (33) and (35)).

- in the general case, the system of equations Eqs. (34), (35) must be solved numerically and these results are discussed in the following sections.

\section{Results}

Our study will examine the instability dependence on 4 parameters: 1) the fluid velocity $\beta$ (alternatively, the Lorentz factor $\gamma$ ); 2) the relativistic Mach number

$\mathcal{M}_{\mathrm{r}}=\frac{\beta}{C_{\mathrm{s}}} \frac{\sqrt{1-C_{\mathrm{s}}^{2}}}{\sqrt{1-\beta^{2}}} ;$

3) the Alfvénic Mach number $\zeta=V_{\mathrm{A}} / C_{\mathrm{s}}$; and 4) the ratio $f=m / k$, that indicates the angle between the wave number projection on the $x z$ plane and the flow velocity. In general, relativistic effects come into play whenever one of $\beta, \mathcal{M}_{\mathrm{r}}$, or $\zeta$ (or a combination of them) describes situations of high fluid velocities, hot gas, or strong magnetic field, respectively. To this purpose, it is useful to use the explicit relation linking the Alfvèn speed and other parameters:

$V_{\mathrm{A}}=\frac{\beta \zeta}{\sqrt{\beta^{2}+\mathcal{M}_{\mathrm{r}}^{2}\left(1-\beta^{2}\right)}} \cdot$

In the following, we discuss the non-relativistic case and subsequently the relativistic case, both for oblique propagation $(f \neq 0)$ and for propagation parallel to the velocity interface $(f=0)$. The latter case is important in comparing with results obtained by previous investigators.

\subsection{Non-relativistic flows}

As a starting point, we consider the case of a non-relativistic fluid with $\beta=10^{-3}$ and $\Gamma=5 / 3$.

To gain a comprehensive view of the instability behavior, when one allows for non-parallel propagation (i.e. $f \neq 0$, see also Gerwin 1968; Choudhury \& Lovelace 1986), we represent the results on a contour plot such as that in Fig. 1, where the contour levels show the growth rate $\operatorname{Im}(\phi)$ as a function of $f$ and $\mathcal{M}_{\mathrm{r}}$, for fixed Alfvèn Mach number $\zeta=1.6$ (top panel). From the plot, we can observe that i) two separate instability regions are present in the plane $\left(\mathcal{M}_{\mathrm{r}}, f\right)$, corresponding to slow and fast magnetosonicm unstable modes; and ii) they do not extend to the horizontal axis, i.e. at $f=0$. By expanding the solution of the dispersion relation in terms of $1 / f$, we can show from Eqs. (34), (35) that the corresponding first order term of the growth rate can be expressed by:

$\phi \sim \frac{1}{f}\left[\frac{V_{\mathrm{A}}^{2}-\beta^{2}}{1-\beta^{2} V_{\mathrm{A}}^{2}}\right]^{\frac{1}{2}}$.
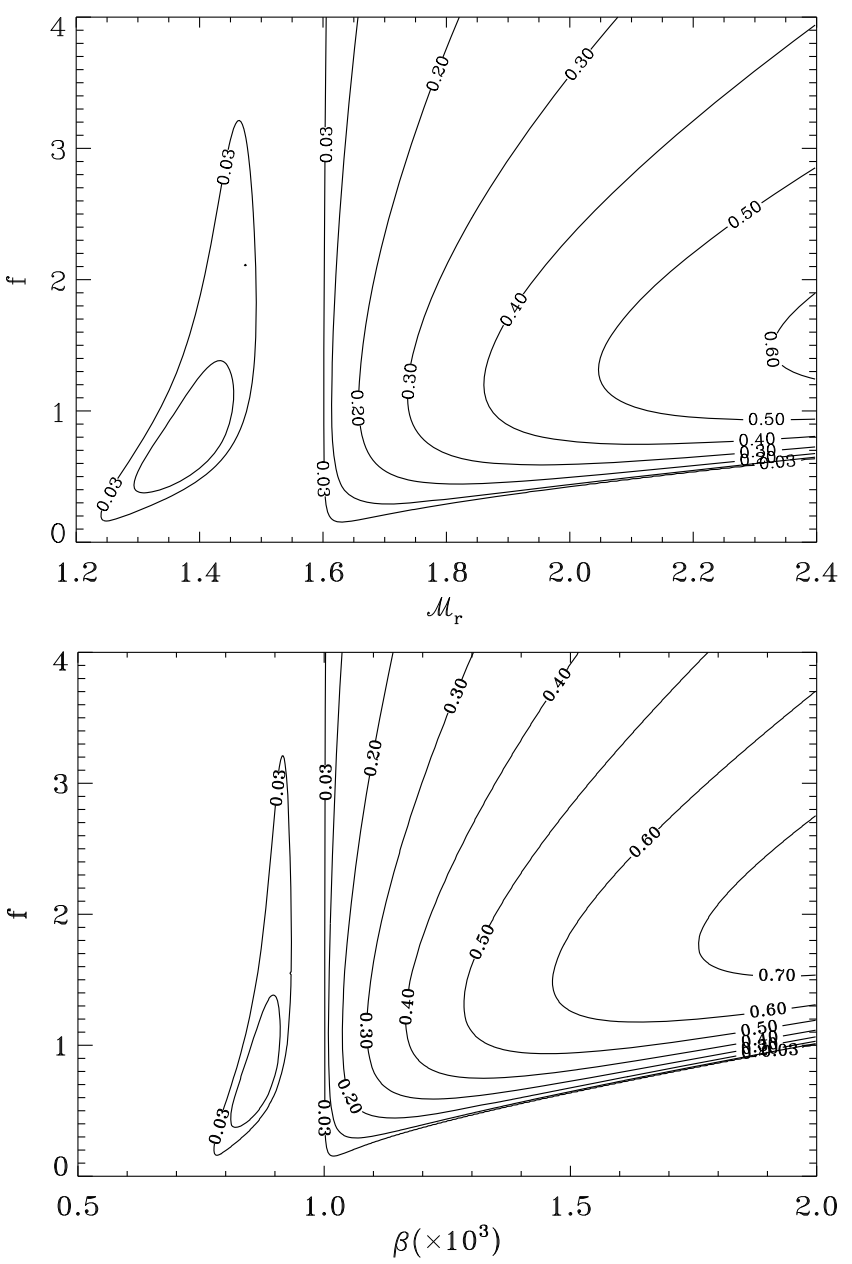

Fig. 1. Two contour plots are shown. Top panel: the contour plot of constant $\operatorname{Im}(\phi)$ surfaces as a function of the inclination factor $f$ and relativistic Mach number $\mathcal{M}_{\mathrm{r}}$ for fixed Alfvénic Mach number $\zeta=1.6$ and flow velocity $\beta=10^{-3}$. Bottom panel: the contour plot of constant $\operatorname{Im}(\phi)$ surfaces as a function of the inclination factor $f$ and $\beta$ for the fixed Alfvén speed $\left(V_{\mathrm{A}}=10^{-3}\right)$ and the sound speed $\left(C_{\mathrm{s}}=6.3 \times 10^{-4}\right)$. Here $\Gamma=5 / 3$ is used.

Equation (39) demonstrates that, for large values of $f$, the instability appears when $V_{\mathrm{A}}<\beta$. In Fig. 1 (bottom panel), we show the contour plot of constant $\operatorname{Im}(\phi)$ surfaces as a function of $f$ and $\beta$ for $V_{\mathrm{A}}=10^{-3}$ and $C_{\mathrm{s}}=6.3 \times 10^{-4}$ (this value corresponds to $\mathcal{M}_{\mathrm{r}}=1.6$ (top panel), see Eq. (37)), and the figure shows that the instability appears for $\beta>10^{-3}$, i.e. when $\beta$ exceeds the value of the Alfvén speed parameter. We note as well that, while the result in Eq. (39) is valid in the non-relativistic limit $\beta \ll 1$, the condition for instability derived above for large $f$ 's can also be written $\zeta<\mathcal{M}_{\mathrm{r}}$, as evident for Fig. 1 (top panel).

For smaller $f$ 's, corresponding to small inclinations of the propagation direction with respect to the velocity interface, Fig. 1 indicates that for $f \lesssim 0.2$ the system becomes stable for the set of parameters considered.

This general behavior is understood more accurately by considering at the real and imaginary parts of the roots that produce unstable modes, shown in Fig. 2. This plot corresponds to a horizontal cut across the contour lines of Fig. 1 for fixed $f=1$. For $\mathcal{M}_{\mathrm{r}}<a$, all roots are real yielding a stable configuration. For $a<\mathcal{M}_{\mathrm{r}}<b$, however, the roots become complex conjugate with nonzero imaginary parts, which make this range unstable. Between $b<\mathcal{M}_{\mathrm{r}}<c$, we have a stable configuration, whereas 


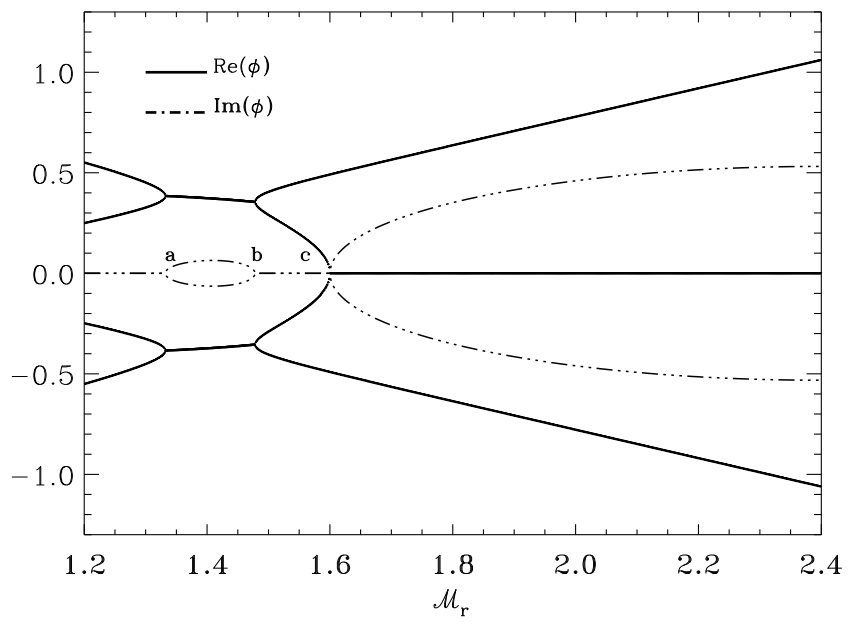

Fig. 2. Imaginary (dotted-dashed lines) and real (solid lines) parts of the roots satisfying the dispersion relation as functions of the relativistic Mach number. Only roots associated with unstable modes are plotted. For the sake of clarity, unphysical roots have been plotted as well. The other adopted set is: $\beta=10^{-3}, \zeta=1.6, f=1$ and $\Gamma=5 / 3$. Instability arises when $a<\mathcal{M}_{\mathrm{r}}<b$ and $\mathcal{M}_{\mathrm{r}}>c$.

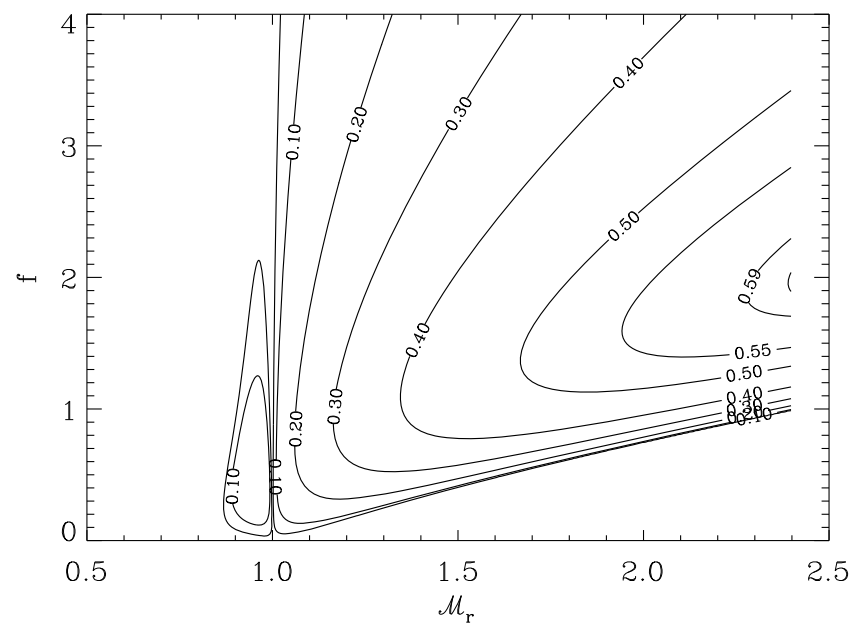

Fig. 3. Same as Fig. 1, but for $\zeta=1$.

for $\mathcal{M}_{\mathrm{r}}>c$ the roots become purely imaginary and a second unstable region can be recognized. By direct substitution into Eqs. (22) and (23) it can be verified that the two family of solutions correspond separately to the onset of slow (for $a<\mathcal{M}_{\mathrm{r}}<b$ ) and fast $\left(\mathcal{M}_{\mathrm{r}}>c\right)$ magnetosonic modes, respectively.

For decreasing magnetization (i.e. lower values of $\zeta$ ), the two instability regions approach each other, gradually shifting towards lower values of $\mathcal{M}_{\mathrm{r}}$. At the same time, lower values of $f$ become prone to instability (see Fig. 3). The limiting case $f=0$ is reached for $\zeta<1$, when the previously identified instability ranges merge into a single unstable region.

We now examine the case of parallel propagation $(f=0)$ for different values of $\zeta<1$. These results may be compared directly with those obtained by Ferrari et al. (1980). In Fig. 4, we plot the growth rate as a function of $\mathcal{M}_{\mathrm{r}}$ for several values of the Alfvén Mach number in the range $0 \leq \zeta \leq 0.9$. For vanishing magnetic field $(\zeta=0)$, the instability disappears when $\mathcal{M}_{\mathrm{r}}>\sqrt{2}$, which is consistent with the results of Bodo et al. (2004). As the magnetic field increases, higher values of $\zeta$ induce a stabilizing effect by raising the pressure and forcing the flow to be channeled along the field lines. This has two noticeable effects: one is to decrease

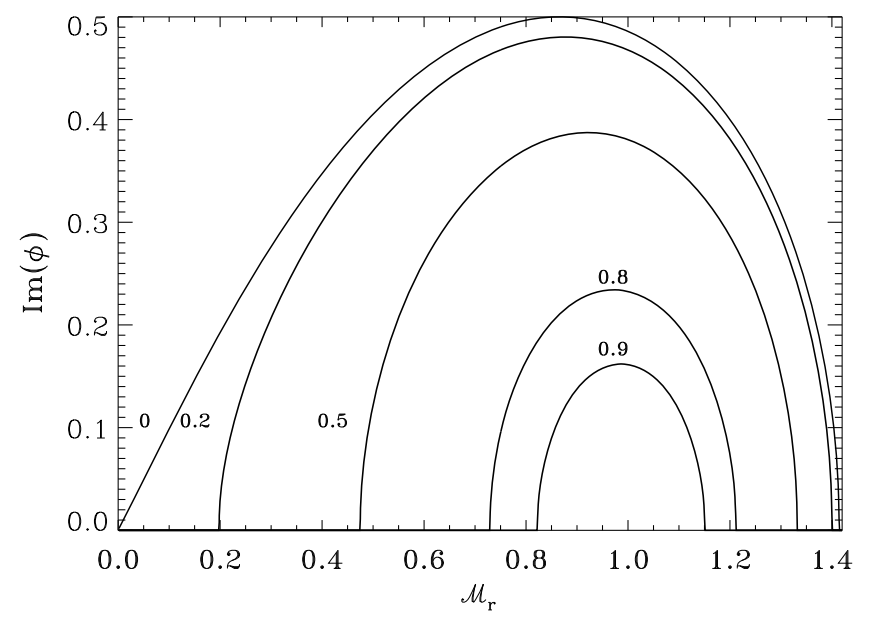

Fig. 4. Dependence of $\operatorname{Im}(\phi)$ on the relativistic Mach number for fixed $\beta=10^{-3}$ and $f=0(\Gamma=5 / 3)$. The different labels above each curve indicate the values of the Alfvénic Mach number $\zeta=[0,0.2,0.5,0.8,0.9]$.

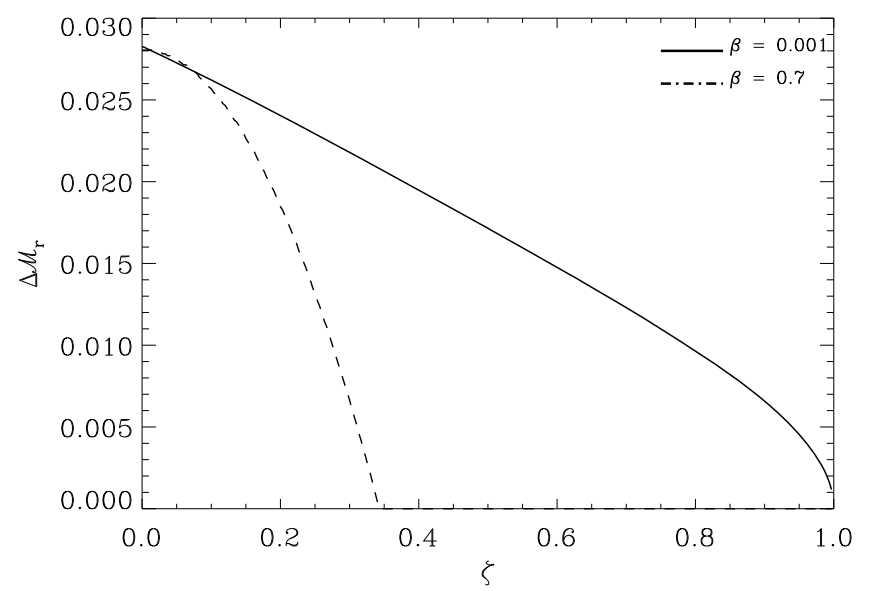

Fig. 5. Instability Mach range $\Delta \mathcal{M}_{\mathrm{r}}$ as a function of the magnetic field in the case of parallel propagation for $\beta=10^{-3}$ (solid line, $\Gamma=5 / 3$ ) and $\beta=0.7$ (dashed line, $\Gamma=4 / 3$ ). For the sake of comparison, the latter has been reduced by a factor of 50. Clearly, the instability is suppressed when the Alfvénic Mach number equals 1 (for $\beta=10^{-3}$ ) and 0.35 (for $\beta=0.7)$.

the maximum value attained by the growth rate and the other is todecrease the instability Mach range $\Delta \mathcal{M}_{\mathrm{r}} \equiv \mathcal{M}_{\mathrm{r}}^{\max }-\mathcal{M}_{\mathrm{r}}^{\min }$, where $\mathcal{M}_{\mathrm{r}}^{\max }\left(\mathcal{M}_{\mathrm{r}}^{\min }\right)$ is the highest (lowest) value of the Mach number above (below) which the configuration is stable. We note that the values of the sound speed at the growth rate maxima are not relativistic.

Figure 5 indicates the dependence of the critical range of relativistic Mach number on the magnetic field (solid line). As is clear from the plot, when $\zeta \rightarrow 1$, the relativistic Mach number range tends to zero and the instability disappears. For $\zeta>1$ the interface is always stable. Our results therefore agree fully with those of Ferrari et al. (1980) for the same parameter range $\left(\beta=10^{-3}, f=0\right)$.

\subsection{Relativistic flows}

In the previous subsection, all relevant quantities described flows in non-relativistic conditions. We now consider fluids that exhibit relativistic behavior, at least in some parameter range.

The top panel in Fig. 6 shows the contour levels of the growth rate for fixed flow velocity $\beta=0.7$ and Alfvénic Mach number 

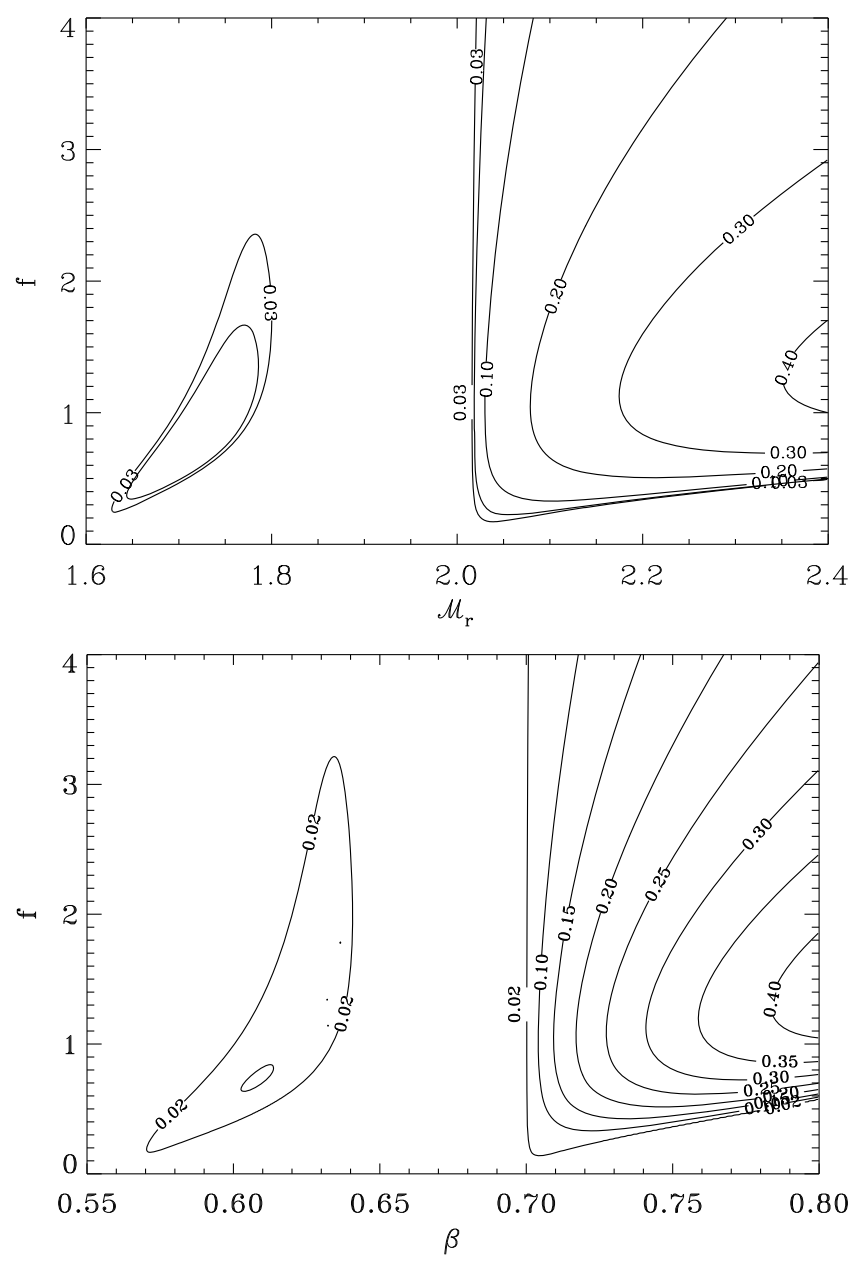

Fig. 6. Top panel: contour plots of constant $\operatorname{Im}(\phi)$ lines in the $\mathcal{M}_{\mathrm{r}}-f$ plane for $\beta=0.7, \zeta=1.6$. The two instabilities branches are clearly visible. Bottom panel: contour plots of constant $\operatorname{Im}(\phi)$ lines in the $\beta-f$ plane for $V_{\mathrm{A}}=0.7, C_{\mathrm{s}}=0.43$. Here $\Gamma=4 / 3$ was used.

$\zeta=1.6$. We have again a slow magnetosonic mode instability region for $1.63<\mathcal{M}_{\mathrm{r}}<1.8$, and a fast magnetosonic mode instability one for $\mathcal{M}_{\mathrm{r}}>2.02$. For large $f$ 's the latter one only survives and the discussion for the non-relativistic case is still valid, i.e. the instability condition is $V_{\mathrm{A}}<\beta$ (see Fig. 6, bottom panel). In fact, a direct comparison between Figs. 6 and 1 reveals a similar qualitative behavior between the two instability regions corresponding to slow and fast magnetosonic modes. In the relativistic case, higher values of $\mathcal{M}_{\mathrm{r}}$ are necessary to promote instability and the gap separating the two regions becomes larger. However, for lower values of $\zeta$, only one instability region survives and there are no contour lines for $\mathcal{M}_{\mathrm{r}}<1.4$ (see the top panel in Fig. 7). This is a consequence of the fact that higher values of the relativistic Mach number correspond to lower sound speeds and, since the latter cannot exceed the upper limiting value of $1 / \sqrt{3}, \mathcal{M}_{\mathrm{r}}$ has a lower physical cutoff at $\sqrt{2} \beta \gamma \simeq 1.386$. No merging can therefore occur as $\zeta$ decreases, since the leftmost region disappears below this threshold. This effect is clearly visible in Fig. 7. In the limit of parallel propagation $(f=0)$, the growth rate decreases with stronger magnetization (see Fig. 8). This is also manifested by the reduced range of Mach number values for which instability exists. This behavior was discussed for non-relativistic flows in Sect. 3.1, where the flow was shown to become stable for values of the Alfvén speed close to the speed of sound. For the present case $(\beta=0.7)$,
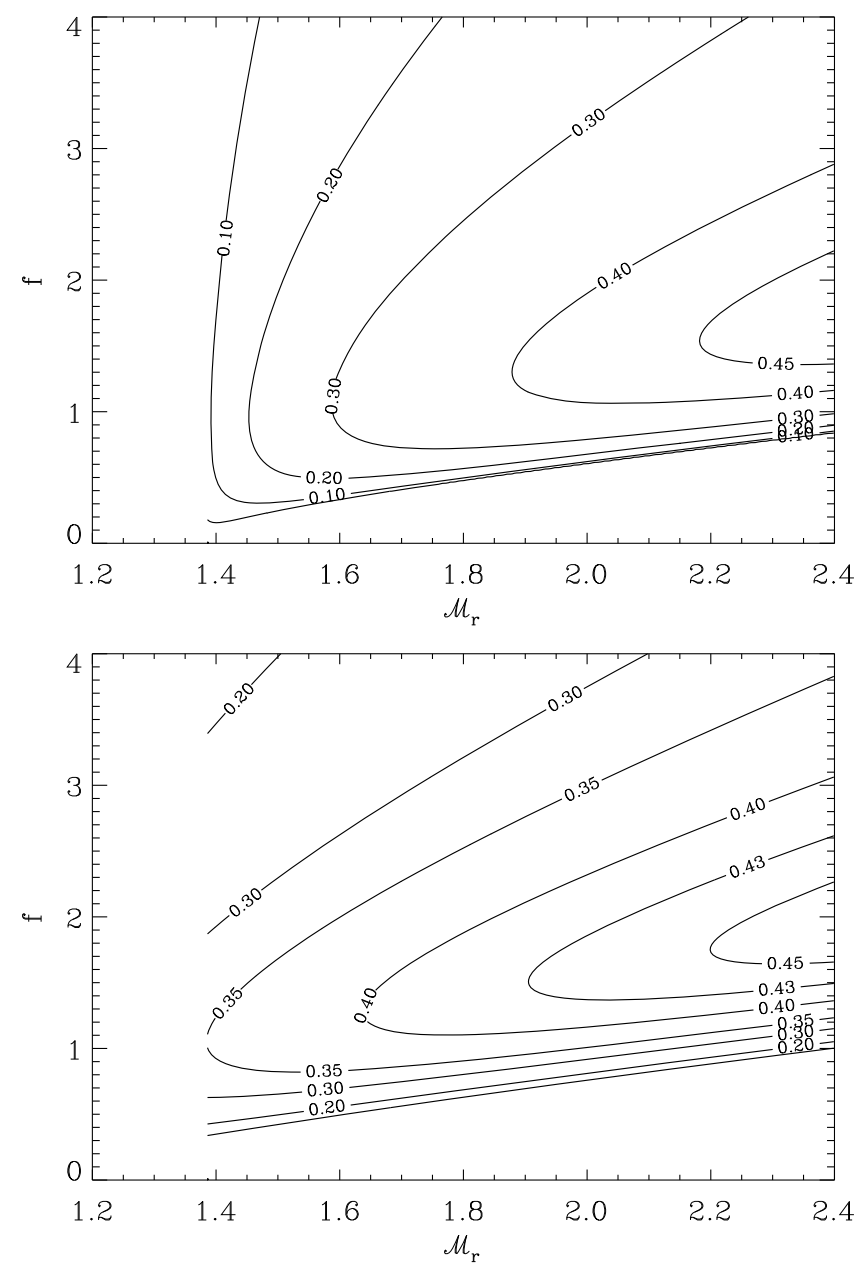

Fig. 7. Contour plots of constant $\operatorname{Im}(\phi)$ lines in the $\mathcal{M}_{\mathrm{r}}-f$ plane for $\beta=0.7, \zeta=1.2$ (top panel) and $\zeta=1$ (bottom panel). Here $\Gamma=4 / 3$ was used.

however, stability is approached when the Alfvén speed $V_{\mathrm{A}} \simeq$ $0.35 C_{\mathrm{s}}$ (see Fig. 8). This tendency can also be recognized from the profile of $\Delta \mathcal{M}_{\mathrm{r}}$ (the critical Mach number instability range) as function of $\zeta$, shown in Fig. 5 (dashed line). As anticipated, the instability is quenched for $\zeta \simeq 0.35$, an effect which can also be justified by the increased kinematic inertia.

These results confirm the general trend already discussed by Ferrari et al. (1980); however, differences in the growth rates are found when the Alfvèn velocity approaches the velocity of light and displacement currents become relevant.

In Fig. 9, we show the critical value of $\zeta$ (above which instability is suppressed) as a function of the flow velocity $\beta$. We can clearly see that $\zeta_{\text {cr }}$ monotonically decreases from $\sim 1$ (at $\beta=0.6$ ) to 0 for $\beta \simeq 0.71$. Higher values of $\beta$ lead to a stable interface, even without magnetic fields. This differs significantly from the classical MHD case. The stabilizing effect of highly relativistic flows was already discussed by Bodo et al. (2004), where it was shown that positive growth rates are subject to the condition

$\beta<C_{\mathrm{s}}\left[\frac{2}{1+C_{\mathrm{s}}^{2}}\right]^{\frac{1}{2}}$.

Since the right-hand side is a monotonically increasing function of $C_{\mathrm{s}}$, we conclude that for $\beta \geq 0.7071\left(C_{\mathrm{s}}=1 / \sqrt{3}\right)$ the instability is suppressed by kinematic effects only, independently of the value of the magnetic field. 


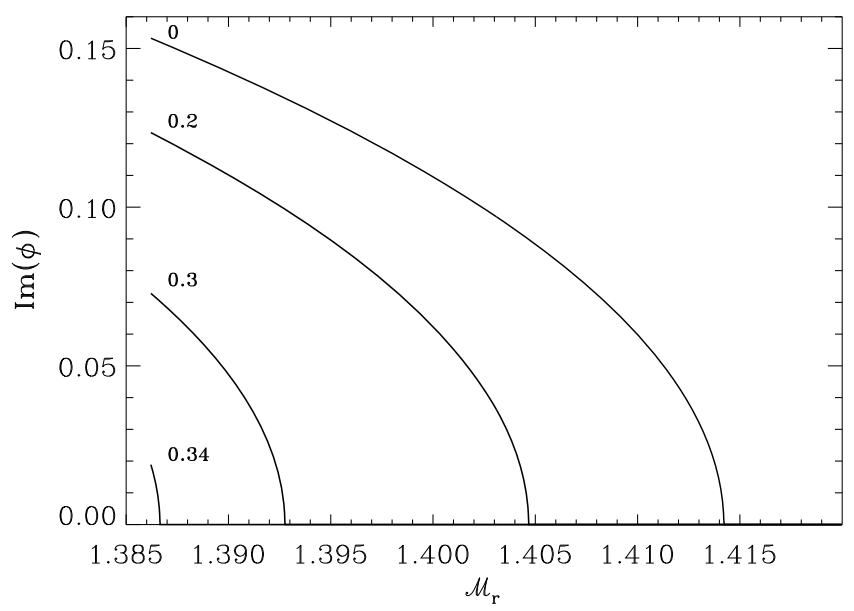

Fig. 8. Effects of magnetic field on the growth rate. Labels on each curve indicate values of $\zeta$. The set of parameters is: $\beta=0.7, \zeta=$ $[0,0.2,0.3,0.34], f=0$ and $\Gamma=4 / 3$. For $\zeta=0.35$, the flow is completely stabilized.

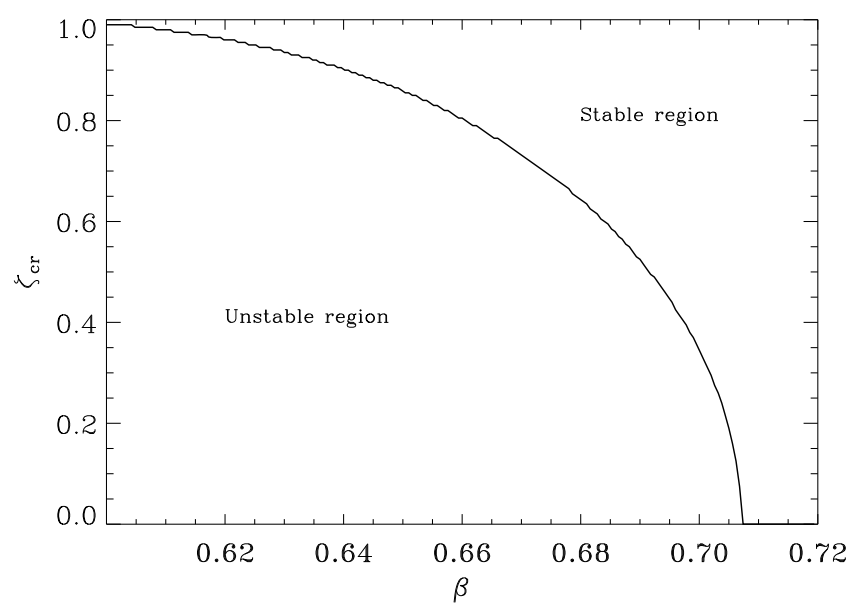

Fig. 9. Effect of the kinematic factor on the instability when $f=0$ ( $\Gamma=4 / 3$ ). As is clear, for $\beta \simeq 0.71$ the instability is suppressed by relativistic effects independently of the value of $\mathcal{M}_{\mathrm{r}}$.

\section{Summary}

We have completed a linear stability analysis of the relativistic, magnetized, Kelvin-Helmholtz instability problem in the vortex sheet approximation. Solutions to the dispersion relation have been sought in terms of four parameters, which provide the strength of the magnetic field, the relativistic Mach number, the flow velocity, and the spatial orientation of the wave vector. The main results of this analysis can be summarized as follows:

1. We have examined the cases of non-relativistic and relativistic flows separately. We have found that, for fixed values of the Alfvèn velocity, two separate regions of instability appear in the plane $\left(\mathcal{M}_{\mathrm{r}}, f\right)$. These two instability regions are associated with the destabilization of slow and fast magnetosonic modes. For large $f$ 's, only the fast magnetosonic modes are unstable in the region $V_{\mathrm{A}}<\beta$.

2. For high values of the Alfvèn velocity, modes propagating parallel to the flow velocity are stable. As this velocity decreases, the two instability regions, corresponding to slow and fast magnetosonic modes, tend to merge and the parallel modes eventually destabilize. This general behavior is common for both non-relativistic and relativistic flows.
3. We have found that slow modes non-parallel to the flow direction are gradually excluded from the instability plane as they become unphysical. This effect takes place at increasingly relativistic velocities or low magnetic fields or a combination of both.

4. When considering the case of parallel propagation $(f=0)$, we have found that, similar to the non-magnetic counterpart, the flow becomes linearly stable when the relativistic Mach number exceeds a critical value. In the limit of a vanishing Lorentz force, this threshold reaches the maximum value of $\sqrt{2}$, in a frame where the fluids have equal and opposite velocities.

5. For increasing magnetic field strength, the maximum unstable growth rate decreases and the instability exists for a narrow range of Mach number values.

6. The main difference from the non relativistic MHD is that, at higher flow velocities (for $f=0$ ), kinematic effects stabilize the flow even for smaller values of the Alfvén velocity. Furthermore, the computed growth rates attain lower values than their classical counterparts. When the flow velocity becomes higher than $1 / \sqrt{2}$, the flow does not require the magnetic field for stabilization.

Relativistic flows stabilize the KH modes due to the concurrence of two effects First, at high relative velocities, the mode coupling between the two half-spaces loosens because of causality effects; this effect is present in the non-relativistic case as well but is even more relevant at relativistic velocities Second, in the relativistic regime the inertia of the fluid particles is augmented dynamically, hampering the response of the fluid to the instability.

Destabilization of relativistic Kelvin-Helmholtz modes has been applied in the astrophysical context of jets of extended extragalactic radio sources and relativistic galactic sources to explain the generation of observed morphologies and the driving mechanisms for their radiation emission. While originally these modes were thought to disrupt the collimated propagation, it was realized later that the head of jets creates a bow shock and a cocoon that shield the flow from steep gradient boundary layers, while leading to generation of a turbulent state. Turbulence is functional in producing mixing of jet and ambient matter with entrainment, angular momentum transport and deceleration of the flow (Rossi et al. 2008). The behavior of KHI as a function of the physical parameters is likely to the at the base of the different classes of radio sources. In addition, long wavelength modes may develop and generate global structures such as the wiggles and knots that are observed in astrophysical jets. Our fully relativistic analysis has confirmed that to activate $\mathrm{KH}$ modes small $V_{\mathrm{A}} / C_{\mathrm{S}}$ ratios are required. This condition can be achieved in very hot plasmas, even for relatively large magnetic fields, as appears to be the case in high-energy sources.

$\mathrm{KHI}$ also appears to be relevant in the case of PWNe; in these objects, a relativistically hot magnetized plasma is produced by the pulsar ultra-relativistic wind and interacts with the surrounding supernova ejecta. These PWNe are internally structured and have high velocity flow channels that can become KH unstable. As already mentioned, Bucciantini \& Del Zanna (2006) examined numerically the nonlinear development of KHI in these channels, assuming planar geometry, to interpret the signature of the instability on the synchrotron emission. The present linear stability analysis is complementary to their study because it allows more accurate comprehension of the physical phenomena involved in the destabilization process. Again the presence of a hot plasma appears necessary to start the channel destabilization. 
Acknowledgements. The authors are grateful to Dr. Edoardo Trussoni for valuable discussions. The research was partially supported by the Georgian National Science Foundation grant GNSF/ST06/4-096.

\section{References}

Anile, A. M. 1989, Relativistic fluids and Magneto-Fluids (Cambridge: Cambridge Univ. Press)

Blandford, R., \& Pringle, J. 1976, MNRAS, 176, 443

Bodo, G., Rossi, P., Mignone, A., Massaglia, S., \& Ferrari, A. 2003, New Astron. Rev., 47, 557

Bodo, G., Mignone, A., \& Rosner, R. 2004, Phys. Rev. E, 70, 036304

Bucciantini, N., \& Del Zanna, L. 2006, A\&A, 454, 393

Chandrasekhar, S. 1961, Hydrodynamic and Hydromagnetic Stability (Oxford: Clarendon)

Hardee, P. E. 1987, ApJ, 313, 607

Hardee, P. E. 2007, ApJ, 664, 26

Ferrari, A., Trussoni, E., \& Zaninetti, L. 1980, MNRAS, 193, 469
Ferrari, A., Trussoni, E. \& Zaninetti, L. 1981, MNRAS, 196, 1051

Ferrari, A., Massaglia, S., \& Trussoni, E. 1982, MNRAS, 198, 1065

Gerwin, R. A. 1968, Rev. Mod. Phys., 40, 652

Komissarov, S. S. 1999, MNRAS, 303, 343

Königl, A. 1980, Phys. Fluids, 23, 1083

Lord, K. 1871, Philos. Mag., 42, 362

Lichnerowicz, A. 1967, Relativistic Hydrodynamics and Magnetohydrodynamics (New York: Benjamin)

Mirabel, I. F., \& Rodriguez, L. F. 1999, ARA\&A, 37, 409

Pu, Z. Y., \& Kivelson, M. G. 1983, J. Geophys. Res., 88, 841

Roychoudhury, S., \& Lovelace, R. V. E. 1986, ApJ, 302, 188

Rossi, P., Bodo, G., Massaglia, S., Ferrari, A., \& Mignone, A. 2004, Ap\&SS, 293, 149

Rossi, P., Mignone, A., Bodo, G., Massaglia, S., \& Ferrari, A. 2008, A\&A, 488, 795

Sen, A. K. 1963, Phys. Fluids, 6, 1154

Sen, A. K. 1963, Phys. Fluids, 7, 1293

Turland, B. D., \& Scheuer, P. A. G. 1976, MNRAS, 176, 421

Von Helmholtz, H., \& Monats, K. 1868, Preuss. Akad. Wiss. Berlin, 23, 215 